\title{
Evaluation of Deep Learning-based Approaches to Segment Bowel Air Pockets and Generate Pelvis Attenuation Maps from CAIPIRINHA- accelerated Dixon MR Images
}

Hasan Sari ${ }^{1}$, Ja Reaungamornrat ${ }^{2}$, Onofrio A. Catalano ${ }^{1}$, Javier Vera-Olmos ${ }^{3}$, David Izquierdo-Garcia ${ }^{1}$, Manuel A. Morales ${ }^{1}$, Angel Torrado-Carvajal ${ }^{1,3}$, Thomas S.C. $\mathrm{Ng}^{4}$, Norberto Malpica ${ }^{3}$, Ali Kamen², and Ciprian Catana ${ }^{1}$

1. Athinoula A. Martinos Center for Biomedical Imaging, Massachusetts General Hospital and Harvard Medical School, Charlestown, MA, United States

2. Siemens Corporate Research, Princeton, NJ, United States

3. Medical Image Analysis and Biometry Lab, Universidad Rey Juan Carlos, Madrid, Spain

4. Department of Radiology, Massachusetts General Hospital, Boston, MA, United States

Corresponding author: Ciprian Catana, Athinoula A. Martinos Center for Biomedical Imaging, Massachusetts General Hospital and Harvard Medical School, Charlestown, MA, United States. Tel: +1(617)-643-4885, e-mail: ccatana@mgh.harvard.edu Running Title: DL-based AC for Pelvis PET/MR imaging Word Count: 5303 words Abstract Word Count: 277 


\section{ABSTRACT}

Attenuation correction (AC) remains a challenge in pelvis PET/MR imaging. In addition to the segmentation/model-based approaches, deep learning methods have shown promise in synthesizing accurate pelvis attenuation maps ( $\mu$-maps). However, these methods often misclassify air pockets in the digestive tract, which can introduce bias in the reconstructed PET images. The aims of this work were to develop deep learning-based methods to automatically segment air pockets and generate pseudo-CT images from CAIPIRINHA-accelerated MR Dixon images. Methods: A convolutional neural network (CNN) was trained to segment air pockets using 3D CAIPIRINHA-accelerated MR Dixon datasets from 35 subjects and was evaluated against semi-automated segmentations. A separate CNN was trained to synthesize pseudo-CT $\mu$-maps from the Dixon images. Its accuracy was evaluated by comparing the deep learning-, model- and CT-based $\mu$-maps using data from 30 of the subjects. Finally, the impact of different $\mu$-maps and air pocket segmentation methods on the PET quantification was investigated. Results: Air pockets segmented using the CNN agreed well with semi-automated segmentations, with a mean Dice similarity coefficient of 0.75 . Volumetric similarity score between two segmentations was $0.85 \pm 0.14$. The mean absolute relative change (RCs) with respect to the CT-based $\mu$-maps were $2.6 \%$ and $5.1 \%$ in the whole pelvis for the deep learning and model-based $\mu$-maps, respectively. The average RC between PET images reconstructed with deep learning and CT-based $\mu$-maps was $2.6 \%$. Conclusion: We presented a deep learningbased method to automatically segment air pockets from CAIPIRINHA-accelerated Dixon images with comparable accuracy to semi-automatic segmentations. We also showed that the $\mu$-maps synthesized using a deep learning-based method from CAIPIRINHA- 
accelerated Dixon images are more accurate than those generated with the model-based approach available on integrated PET/MRI scanner.

Keywords: Attenuation correction, PET/MR, PET quantification, deep learning, pseudoCT 


\section{INTRODUCTION}

Accurately accounting for annihilation photons attenuation is essential for quantitative positron emission tomography (PET). In integrated PET and computed tomography (PET/CT) scanners, CT data are scaled to generate attenuation maps ( $\mu$-mapcT) that are used for PET attenuation correction (AC). In integrated PET and magnetic resonance imaging (PET/MRI) scanners, AC has been a challenge as MRI does not directly provide information about tissue attenuation properties (1). The method initially implemented on one of the commercially available PET/MRI scanners, the Biograph mMR scanner (Siemens Healthineers, Erlangen, Germany), segmented the Dixon MR images into four compartments (i.e., background, lung, fat and soft tissue) and assigned known linear attenuation coefficients (LACs) to each of these classes to generate 4-compartment segmented $\mu$-maps ( $\mu$-mapmR4C) (2). As properly accounting for bone tissue attenuation is important, particularly in the pelvis, a model-based approach was subsequently developed to add bone tissue to the $\mu$-mapмR4c. This whole-body 5-compartment modelbased $\mu$-map ( $\mu$-mapmR5c) generation approach uses a database of aligned MR images and bone segmentations for major body bones and involves co-registration of the subject's MR image to the MR model $(3,4)$. The current method implemented on the Biograph mMR (software version VE11P) leverages the controlled aliasing in parallel imaging results in higher acceleration (CAIPIRINHA)-accelerated Dixon 3D-VIBE sequence to acquire diagnostic quality images with improved spatial resolution within the typical 18 second acquisition. In addition to providing diagnostic quality images, this sequence was previously shown to improve the accuracy of the $\mu$-mapMR4C (5). 
Although the 5-compartment approach reduces the bias in the PET data quantification compared to the 4-compartment approach, it has several limitations when imaging the pelvis, the main focus in this work. First, air pockets (i.e., digestive tract gas) are difficult to identify and segment based on the MRI data, which leads to biased PET data quantification. Second, this $A C$ method is prone to registration errors and does not account for the intra- and inter-subject variability in bone density.

Deep learning-based methods are being rapidly adopted in the medical imaging field with many applications in image segmentation(6-8), image registration $(9,10)$, image classification $(11,12)$, etc. Such approaches that use convolutional neural networks (CNNs) and Generative Adversarial Networks have also been implemented in PET and $\mathrm{PET} / \mathrm{MR}$ imaging for various purposes, including image synthesis of $\mathrm{CT}$ images for PET AC (or radiotherapy planning) (13-15). In the context of pelvis AC, deep learning methods have been used to create synthetic CT images using Dixon MR and proton-densityweighted zero-echo-time $(16)$, standard Dixon $(17,18)$ and T1-weighted LAVA Flex wateronly and T2-weighted MR images (19). All of these studies reported improvements in the accuracy of $\mu$-maps and reduced bias in the reconstructed PET images compared to those obtained using the standard segmentation-based $\mu$-maps.

Previously proposed deep learning-based methods cannot synthesize accurate pseudo-CTs from pelvis MR images is the presence of air pockets as they have similar intensity to bone structures in the standard MR images. Furthermore, perfectly matched CT and MR images required for training CNNs are not available since these images are acquired on separate scanners at different times. Therefore, the locations and sizes of the air pockets change between the two scans, leading to errors in both MR-CT co- 
registration and image synthesis tasks. As an initial solution, Torrado-Carvajal et al. (17) filled the air pockets with values corresponding to soft tissue in the estimated $\mu$-map images. Leynes et al. (16) filled the air pockets present in the CT images with soft tissue Hounsfield unit $(\mathrm{HU})$ values prior to training the CNN model. They reported artefacts in their pseudo-CTs due to air pockets being assigned bone HUs. Both groups excluded the air pocket voxels from the PET data bias analyses. Alternatively, Bradshaw et al. (19) used a technique which involved an intensity-based threshold, morphological closing and manual adjustments to localise air pockets and place them on $\mu$-maps.

In this work, we trained and evaluated CNNs to automatically segment air pockets from Dixon MR images and assessed the quantitative impact on the reconstructed PET images. Furthermore, we used the higher quality CAIPIRINHA-accelerated Dixon images within a deep-learning framework to generate pelvis pseudo-CT maps, compared them to the $\mu$-mapmR5c and $\mu$-mapcr and evaluated the impact of using these $\mu$-maps on PET data quantification. Consideration of bowel gas positioning during the PET data acquisition will likely significantly impact accurate assessment of pelvic lesion uptake, and have potential impactful clinical ramifications in both staging and longitudinal treatment assessment. While outside the scope of the current study, our work therefore provides the technical foundation for future prospective studies to assess the impact of the proposed techniques.

\section{MATERIALS AND METHODS}

This retrospective study included data from 30 oncological patients (age: $57 \pm 10$, 19 females $/ 11$ males, weight $69 \pm 15 \mathrm{~kg}$ ) who underwent successive, same day PET/CT 
(as part of standard care) and PET/MR (research) examinations. CAIPIRINHAaccelerated MR Dixon data acquired from five additional subjects (age: $57 \pm 5,3$ females $/ 2$ males, weight $72 \pm 8 \mathrm{~kg}$ ) were also included in this study and used only in the development and evaluation of the air pocket segmentation method. All patients gave written informed consent, and the local Institutional Review Board approved the study.

PET/MR Data: Simultaneous PET and MR data were acquired using the Biograph mMR scanner. Whole body 18F-FDG PET data were acquired in 4 bed positions (injected dose: $568 \pm 78 \mathrm{MBq}$ ) for 20 minutes approximately 2 hours after radiotracer administration. Whole body MR data were acquired in 4 bed positions using the CAIPIRINHA-accelerated Dixon 3D Vibe sequence (TR: 3.96 ms, TE1: 1.23 ms, TE2: $2.46 \mathrm{~ms}$, FA: $9^{\circ}$, scan duration 18 seconds) approximately 10 minutes after injection of Gadolinium-based MR contrast agent (Dotarem). This sequence provides in-phase, opposed phase, water and fat T1-weighted images that are typically used in the modelbased $\mu$-map estimation method. MR images were reconstructed with a voxel size of $2.1 \times 2.6 \times 2.1 \mathrm{~mm}^{3}$.

CT Data: Low-dose CT data were acquired as part of the PET/CT acquisitions using Discovery 710 (GE Healthcare, WI, USA) $(n=26)$ and Siemens Biograph $64(n=4)$ (Siemens Healthineers, Erlangen, Germany) PET/CT scanners (voltage: $120 \mathrm{kV}$, tube current: $150 \mathrm{~mA}$ ). The CT images were reconstructed with a voxel size of $0.98 \times 0.98 \times 5$ $\mathrm{mm}^{3}$. The CT data obtained from the two scanners were considered equivalent for the purpose of this study.

Image processing: MR images were first corrected for low frequency intensity nonuniformity using N4 bias correction (20). The scanner bed was removed from the whole- 
body CT images using intensity thresholding and morphological operations. Subsequently, the pelvis region was manually cropped from the whole-body images. CT/MR pairs were co-registered using affine and non-rigid transformations using NiftyReg (21). Finally, the images were resampled to generate a volume with $256 \times 256 \times n$ voxels. Voxel size of each volume was approximately $2.0 \times 1.6 \times 2.0 \mathrm{~mm}^{3}$.

\section{Air Pockets Segmentation}

Air pockets present in the CT images were segmented using an image thresholding algorithm (HU values <-700). Air pockets in the MR images were segmented using a region growing algorithm implemented in the ITK SNAP software (22). This semiautomatic procedure required manual placement of seeds on air pockets and editing of the resulting segmentations by experienced radiologists. These air pockets were used to train a CNN. A UNet $(6,23)$ architecture with residual units, consisting of 4 down-sampling and 4 up-sampling layers, with rectified linear unit (ReLU) used as the activation function, was chosen for this task. 3D MR Dixon in-phase volumes were used as input data. The acquired images were resampled to an isotropic volume with voxel size of $1 \mathrm{~mm}^{3}$ and multiple patches with a fixed matrix size of $96 \times 96 \times 96$ voxels were extracted. To avoid overfitting during the training, data augmentation was performed by applying $\pm 10 \%$ image scaling and a random rotation with $\pm 10^{\circ}$ angle. The MR volumes were normalised to zero mean and unity variance. The network was trained and evaluated on a dataset of 35 subjects using a five-fold cross-validation, where for each fold, the data was split into $80 \%$ training (28 subjects) and $20 \%$ validation/testing data (7 subjects). Dice similarity 
coefficient (DSC) was used as the loss function and the network was trained using an Nvidia Tesla V100 GPU.

The accuracy of the segmentation network was evaluated by computing segmented air pocket volumes, DSC, and Hausdorff distance (24) at $95^{\text {th }}$ percentile between the segmentations obtained using the CNN and the semi-automatic methods. DSC and Hausdorff distance are two metrics commonly used in evaluating image segmentation methods and are measures of similarity and largest segmentation error between two segmented regions. Volumetric similarity (VS) (25) was also computed using equation 1:

$$
V S=1-\frac{\left|V-V_{r e f}\right|}{V+V_{r e f}}
$$

where $\mathrm{V}$ is the test volume and $\mathrm{V}_{\text {ref }}$ is the volume of semi-automatically segmented air pockets.

\section{Pelvis Attenuation Map Synthesis}

A separate network also based on the UNet (6) architecture was trained to synthesize pseudo-CT images from the four Dixon 2D axial images (17). Mean absolute error (MAE) was used as the loss function. During the training, data augmentation was performed by applying random displacements of 5 voxels and a random flip in the slices. The Dixon volumes were normalised to have zero mean and unity variance. A five-fold cross-validation was performed where data was split to $80 \%$ training (24 subjects) and $20 \%$ validation/testing data (6 subjects).

The HU values of the output pseudo-CT images were scaled to obtain the $\mu$-maps (26). Voxels belonging to air-pockets were assigned a LAC of zero. $\mu$-mapMR5C and $\mu$ - 
mapcт were also generated. All $\mu$-maps were smoothed using a Gaussian filter with $4 \mathrm{~mm}$ full width at half maximum (FWHM) kernel to match the resolution of the PET images. The percentage absolute and non-absolute relative change $(R C)$ were computed using equation 2:

$$
R C(\%)=100 \frac{\left|I-I_{r e f}\right|}{I_{r e f}}
$$

where $I$ is the test image and $I_{\text {ref }}$ is the reference image. CT based $\mu$-maps with CNNderived air pockets were used as the reference image. Absolute and non-absolute RCs were evaluated voxelwise in the whole pelvis and within 3 ROls: bone, fat- and waterbased soft tissue. These ROls were segmented using a thresholding algorithm on the ground truth $\mu$-mapct. Bones were obtained by excluding voxels with LACs $<0.105 \mathrm{~cm}^{-1}$ and applying a flood-fill operation in order to capture the bone marrow. Water-based soft tissue ROI was obtained by only keeping non-bone voxels within the $0.090-0.105 \mathrm{~cm}^{-1}$ range and fat-based soft tissue ROI was obtained by only selecting voxels with LACs in the $0.080-0.090 \mathrm{~cm}^{-1}$ range.

\section{Impact on PET Data Quantification}

To evaluate the effects of using different $\mu$-map generation methods on PET images, PET image reconstruction was performed using (as also shown in Figure 1):

1. Model-based $\mu$-map with no added air pockets, as generated and used on the Biograph mMR scanner ( $\mu$-mapmR5c).

2. MR and CT-based $\mu$-maps with CNN predicted air pockets from MR Dixon images ( $\mu$-mapMR5C-CNNAIR, $\mu$-mapMRDL-CNNAIR, $\mu$-mapCT-CNNAIR, respectively). 
The PET images were reconstructed with the Siemens e7-tools (version VE11P) using the ordered subsets expectation maximization (OSEM) algorithm ( 3 iterations and 21 subsets), with a voxel size of $2.1 \times 2.1 \times 2.0 \mathrm{~mm}^{3}$ and smoothed using a post-reconstruction Gaussian filter with a FWHM of $4 \mathrm{~mm}$. Absolute and non-absolute percentage RCs between the PET images attenuation corrected using the $\mu$-maps generated with the different methods were computed and reported for the whole pelvis and ROIs listed above. To further study the effects of misclassified air pockets on the PET estimates in adjacent structures, a fourth ROI was obtained by dilating the semi-automatically segmented air pocket masks in all directions by $3 \mathrm{~cm}$ and subtracting the air pocket voxels from the dilated region.

\section{RESULTS}

Example Dixon in-phase MR images with air pockets semi-automatically segmented and predicted by the CNN algorithm are shown in Figure 2. The proposed method was able to segment both large and small volume air pockets, and to distinguish between air pockets and other structures with low signal intensity on MR Dixon in-phase images, particularly bladder and bones, achieving a DSC of $0.75 \pm 0.15$, averaged across the testing/validation folds (Figure 3a). Segmented air pocket volumes for each subject are shown in Figure 3b. The volumetric similarity between the two segmentations was $0.85 \pm 0.14$. Overall, there was no statistically significant difference between the air pocket volumes obtained using the two methods (paired t-test, $p=0.30$ ). Subject 30 had a significantly lower DSC and VS than other subjects. This subject had one of the smallest volumes of air pockets and the CNN misclassified the bladder as air, causing a large 
difference in segmented volumes (Supplementary Figure 1). The average $95^{\text {th }}$ percentile Hausdorff distance between segmentations obtained with each method was $51.0 \pm 52.4$ $\mathrm{mm}$.

The $\mu$-maps generated using model-based method without ( $\mu$-mapмr5c) and with added air pockets ( $\mu$-mapMR5C-CNNAIR), CT ( $\mu$-mapcT-CNNAIR), the deep learning-based method ( $\mu$-mapmRDL-CNNAIR) are shown in Figure 4 for a representative subject. Qualitatively, the deep learning-based method appears to distinguish fat- and waterbased soft tissue more accurately than the model-based method. Better representation of bone structures was also seen in $\mu$-maps generated using the proposed method.

As shown in table 1 , the quantitative assessment confirmed these findings, $\mu$ maPMRDL-CNNAIR being more similar to $\mu$-mapCT-CNNAIR than the $\mu$-mapMR5C-CNNAIR, with lower global and regional RCs. When all the voxels in the pelvis were compared, absolute RC was decreased from $5.1 \%$ to $2.6 \%$ when $\mu$-maps were generated using the deep learning rather than the 5-compartment model-based method. This difference was statistically significant $(p<0.001)$. The largest improvement was seen in the fat soft tissue where the absolute RC was reduced by a factor of 2.6. The difference between absolute and nonabsolute $R C$ values were statistically significant in fat-based soft tissue and water-based soft-tissue ROls. Although there were no significant group differences in the RC values obtained in the bones, the 5-compartment model-based approach failed to assign bone LACs in majority of bones in two subjects (Supplementary Figure 2).

PET images obtained using each $\mu$-map and air pocket segmentation method and the corresponding $\mathrm{RC}$ maps with respect to PETCT-CNNAIR are shown for a representative subject in Figure 5. PETMRDL-CNNAIR had lower global RC values compared to PETMR5C- 
CNNAIR. It can also be seen that PETMR5C had an area under the bladder with significantly higher 18F-FDG uptake compared to the other PET reconstructions. This area corresponds to an air pocket misclassified as soft-tissue in the $\mu$-mapMR5c.

Averaged across all subjects, PETMR5C and PETMR5C-CnNAIR had larger nonabsolute and absolute RC values than PETMRDL-CNNAIR compared to PETCT-CNNAIR, both globally and regionally. Globally, the mean absolute $\mathrm{RC}$ values decreased from $7.1 \%$ to 4.9\%, and to $2.6 \%$ for PETMr5C, PETMR5C-CnNAIR and PETdD-CnNAIR, respectively. As seen

in Figure 6, improvements were also observed in all the ROls. PETMR5C had an RC of $20 \%$ and $12 \%$ in bone regions for the two subjects where model-based $\mu$-maps did not include major bone structures.

In the ROI surrounding air pockets, PETDL-CNNAIR had an absolute RC of $3.0 \pm 1.4 \%$ (range: $0.1 \%-6.2 \%$ ), while PET MR5C had an average absolute RC of $11.0 \pm 6.5 \%$ (range: $0.7 \%-31.6 \%)$. In this ROI, PETMR5C images of two subjects had an absolute RC greater than $22 \%$ as their $\mu$-maps had large volumes of misclassified air pockets near bladder.

\section{DISCUSSION}

Previous studies have highlighted the important role PET (combined with both CT and MRI) plays in staging pelvic malignancies, treatment planning for chemoradiation, and assessment of therapeutic response using multiple different tracers. With the anticipated FDA-approval of PSMA-targeting agents, the clinical need for reliable depiction of pelvic uptake using PET/MRI will only increase. In addition, PET/MRI imaging is actively being explored for evaluation in inflammatory bowel disease (27). For all the applications above, accurate estimation of uptake will likely impact prognosis and choice 
of therapy and treatment response assessment, therefore motivating our current study. First, misclassifying the air pockets as soft tissue could lead to false positives due to overestimation of PET activity in these voxels. Second, lesions with increased uptake located in the vicinity of air pockets could be missed due to the decreased lesion-tobackground contrast. Third, the bias introduced in adjacently located lesions could impact the assessment of longitudinal changes. Finally, from a methodological perspective, completely separating the air pocket segmentation from the pelvis attenuation map generation tasks when using deep learning approaches, might increase the performance of the latter techniques as the related anatomical mismatches between the MR and CT images used for training could be eliminated (i.e., by filling the air pockets with soft tissue in both datasets).

The first aim of this work was to develop a deep learning-based approach to automatically segment air pockets present in the pelvis region from high resolution CAIPIRINHA-accelerated Dixon MR images. Semi-automatic segmentation of air pockets is a laborious and subjective process especially when additional manual editing is required. The proposed CNN trained using semi-automatically segmented air pockets was able to accurately predict air pocket in new datasets with an average DSC of 0.75 , suggesting it could be used to minimize this source of bias in the reconstructed PET images. Our results also showed that misclassifying air pockets as soft tissue can introduce bias in the reconstructed PET images, particularly in the adjacent structures, which could interfere with the clinical interpretations (28).

High resolution CAIPIRINHA-accelerated Dixon in-phase images were used in the delineation of air pockets to provide ground-truth data. However, these images contain 
similar signal in air pockets and some other structures such as bones, spinal cord and some ligaments. Moreover, some of the subjects had a high number of small air pockets trapped between faeces that were missed in the semi-automatic segmentation step but correctly identified by the CNN. Furthermore, CAIPIRINHA-accelerated Dixon in-phase images were acquired approximately 10 minutes after administration of Gadoliniumbased contrast agent, which caused the bladder to be separated into bright and dark areas, the latter being incorrectly classified as large air pockets in some subjects by the CNN. Acquisition of CAIPIRINHA-accelerated Dixon images before contrast agent administration will eliminate this issue and can potentially improve the performance of the air pocket segmentation method. While these infrequent outliers could be corrected during the quality control step, the performance of the proposed method would likely increase if a larger number of datasets were available for training. In principle, this could be explored in future studies using only MR data. The segmentation method proposed could be combined with any pelvis $\mu$-map generation approach to generate maps that accurately reflect the physiological state during the PET data acquisition.

Finally, although PET and MR images were acquired in a single scan using an integrated PET/MR scanner, air pockets could have moved during the data acquisition due to peristalsis. In this study, we segmented the air pockets from a single CAIPIRINHAaccelerated Dixon acquisition and used the resulting $\mu$-maps to attenuation correct the PET data collected over a longer duration. One way to address this potential issue could be repeating the CAIPIRINHA-accelerated Dixon acquisitions to detect potential air pocket movements over the course of the PET/MR scan. 
A second aim of this work was to train and test a separate CNN to generate more accurate pelvis $\mu$-maps than those generated using the approach currently available on the Biograph mMR scanner ( $\mu$-mapMR5c). Qualitative and quantitative analyses indicate a CNN trained with CAIPIRINHA-accelerated Dixon MR images is able to generate $\mu$-maps with a better resemblance to $\mu$-mapcr than $\mu$-mapmR5c. We noticed the overall absolute $\mathrm{RC}$ in the pelvis was reduced by a factor of 2 , which was a similar improvement to that reported by Leynes et al. (16), Torrado-Carvajal et al. (17), and Pozaruk et al. (18). Compared to previous findings, we observed reduced differences in bony regions between the deep learning and model-based $\mu$-maps. This was due to the fact that the bone tissue is no longer misclassified as soft tissue in the $\mu$-mapMR5c generated using the most recent method available on the Biograph mMR scanner.

The proposed image synthesis method uses a supervised CNN to perform a voxelto-voxel regression of MR intensities to CT HU values. This approach assumes perfect registration between the MR and $\mathrm{CT}$ images which is hard to achieve. Our MR and CT data were acquired on different scanners with differences in patient positioning, particularly in thighs flexion and rotation. Although we have used a combination of affine and non-rigid transformations to coregister the MR and CT data of the training and validation datasets, some registration errors might still be present. Unsupervised learning techniques, such as CycleGAN network incorporating cycle consistency loss function (2931), can be used to alleviate the need for perfect alignment of MR-CT pairs. However, these methods require access to larger pools of data for training and they have to be properly validated for AC of PET data. 


\section{CONCLUSIONS}

We presented a deep learning-based method to automatically segment air pockets from CAIPIRINHA-accelerated MR Dixon images. We also showed that a deep learningbased method can be used to synthesize $\mu$-maps more similar to reference CT based $\mu$ maps than the ones generated with the 5-compartment model-based approach as implemented commercially. Although our results suggest this method might improve the confidence intervals in studies requiring the use of quantitative PET metrics, additional studies in patients with pathological changes are required to demonstrate its clinical utility.

\section{DISCLOSURE}

This work was supported in part by the National Institutes of Health grant number R01CA218187. Ja Reaungamornrat and Ali Kamen are full time employees of Siemens Healthineers. No other potential conflicts of interest relevant to this article exist. 


\section{KEY POINTS}

Question: Can we use CAIPIRINHA-accelerated Dixon MR images to automatically segment air pockets in the pelvis area and synthesize accurate pseudo-CT images for attenuation correction of PET data?

Pertinent Findings: A convolutional network to segment air pockets was trained and evaluated using CAIPIRINHA-accelerated Dixon images of 35 subjects. A separate network to synthesize pseudo-CT images was trained and tested using the Dixon images of 30 subjects who underwent sequential PET/CT and PET/MR examinations. In a region surrounding the air pockets, an improvement by a factor of 3.7 was observed when PET images were reconstructed using deep learning-based $\mu$-maps instead of standard model-based $\mu$-maps.

Implications for Patient Care: The proposed deep learning-based method can be used to accurately generate $\mu$-maps with air pockets and can reduce the PET estimation bias in the regions surrounding air pockets. 


\section{REFERENCES:}

1. Catana C. Attenuation correction for human PET/MRI studies. Physics in Medicine and Biology. 2020;65:[Epub ahead of print].

2. Martinez-Moller A, Souvatzoglou M, Delso G, et al. Tissue classification as a potential approach for attenuation correction in whole-body PET/MRI: evaluation with PET/CT data. J Nucl Med. 2009;50:520-526.

3. Koesters T, Friedman KP, Fenchel M, et al. Dixon sequence with superimposed modelbased bone compartment provides highly accurate PET/MR attenuation correction of the brain. J Nucl Med. 2016;57:918-924.

4. Paulus DH, Quick HH, Geppert C, et al. Whole-body PET/MR imaging: quantitative evaluation of a novel model-based MR attenuation correction method including bone. $J$ Nucl Med. 2015;56:1061-1066.

5. Freitag MT, Fenchel M, Bäumer P, et al. Improved clinical workflow for simultaneous whole-body PET/MRI using high-resolution CAIPIRINHA-accelerated MR-based attenuation correction. Eur J Radiol. 2017;96:12-20.

6. Ronneberger O, Fischer P, Brox T. U-net: Convolutional networks for biomedical image segmentation. Lect Notes Comput Sci. 2015;9351:234-241.

7. Li W, Wang G, Fidon L, Ourselin S, Cardoso MJ, Vercauteren T. On the compactness, efficiency, and representation of 3D convolutional networks: brain parcellation as a pretext task. Lect Notes Comput Sci. 2017;10265:348-360.

8. Kamnitsas K, Ledig C, Newcombe VFJ, et al. Efficient multi-scale 3D CNN with fully connected CRF for accurate brain lesion segmentation. Med Image Anal. 2017;36:61-78.

9. de Vos BD, Berendsen FF, Viergever MA, Sokooti H, Staring M, Išgum I. A deep learning framework for unsupervised affine and deformable image registration. Med Image Anal. 2019;52:128-143.

10. Balakrishnan G, Zhao A, Sabuncu MR, Guttag J, Dalca A v. VoxelMorph: a learning framework for deformable medical image registration. IEEE Trans Med Imaging. 2019;38:1788-1800.

11. Ding Y, Sohn JH, Kawczynski MG, et al. A deep learning model to predict a diagnosis of Alzheimer disease by using 18 F-FDG PET of the brain. Radiology. 2019;290:456-464.

12. Hartenstein A, Lübbe F, Baur ADJ, et al. Prostate cancer nodal staging: using deep learning to predict 68Ga-PSMA-positivity from CT imaging alone. Sci Rep. 2020;10:3398.

13. Liu F, Jang H, Kijowski R, Bradshaw T, McMillan AB. Deep learning MR imaging-based attenuation correction for PET/MR imaging. Radiology. 2018;286:676-684.

14. Dong X, Lei Y, Wang T, et al. Deep learning-based attenuation correction in the absence of structural information for whole-body positron emission tomography imaging. Phys Med Biol. 2020;65:[Epub ahead of print].

15. Maspero M, Savenije MHF, Dinkla AM, et al. Dose evaluation of fast synthetic-CT generation using a generative adversarial network for general pelvis MR-only radiotherapy. Phys Med Biol. 2018;63:185001.

16. Leynes AP, Yang J, Wiesinger F, et al. Zero-echo-time and dixon deep pseudo-CT (ZeDD $\mathrm{CT})$ : direct generation of pseudo-CT images for pelvic PET/MRI attenuation correction using deep convolutional neural networks with multiparametric MRI. J Nucl Med. 2018;59:852-858. 
17. Torrado-Carvajal A, Vera-Olmos J, Izquierdo-Garcia D, et al. Dixon-VIBE deep learning (DIVIDE) pseudo-CT synthesis for pelvis PET/MR attenuation correction. J Nucl Med. 2019;60:429-435.

18. Pozaruk A, Pawar K, Li S, et al. Augmented deep learning model for improved quantitative accuracy of MR-based PET attenuation correction in PSMA PET-MRI prostate imaging. Eur J Nucl Med Mol Imaging. Published online 2020:[Epub ahead of print].

19. Bradshaw TJ, Zhao G, Jang H, Liu F, McMillan AB. Feasibility of deep learning-based PET/MR attenuation correction in the pelvis using only diagnostic MR images. Tomography. 2018;4:138-147.

20. Tustison NJ, Avants BB, Cook PA, et al. N4ITK: Improved N3 bias correction. IEEE Trans Med Imaging. 2010;29(6):1310-1320.

21. Modat M, Ridgway GR, Taylor ZA, et al. Fast free-form deformation using graphics processing units. Comput Meth Prog Bio. 2010;98:278-284.

22. Yushkevich PA, Piven J, Hazlett HC, et al. User-guided 3D active contour segmentation of anatomical structures: significantly improved efficiency and reliability. NeuroImage. 2006;31:1116-1128.

23. Kerfoot E, Clough J, Oksuz I, Lee J, King AP, Schnabel JA. Left-ventricle quantification using residual U-Net. In: Lect Notes Comput Sci. Vol 11395. ; 2019:371-380.

24. Crum WR, Camara O, Hill DLG. Generalized overlap measures for evaluation and validation in medical image analysis. IEEE Trans Med Imaging. 2006;25:1451-1461.

25. Taha AA, Hanbury A. Metrics for evaluating 3D medical image segmentation: analysis, selection, and tool. BMC Med Imag. 2015;15:29.

26. Burger C, Goerres G, Schoenes S, Buck A, Lonn A, von Schulthess G. PET attenuation coefficients from CT images: Experimental evaluation of the transformation of CT into PET 511-keV attenuation coefficients. Eur J Nucl Med Mol Imaging. 2002;29:922-927.

27. Catalano OA, Wu V, Mahmood U, et al. Diagnostic performance of PET/MR in the evaluation of active inflammation in Crohn disease. Am J Nucl Med Mol Imaging. 2018;8:62-69.

28. Lodge MA, Chaudhry MA, Udall DN, Wahl RL. Characterization of a perirectal artifact in 18F-FDG PET/CT. J Nucl Med. 2010;51:1501-1506.

29. Wolterink JM, Dinkla AM, Savenije MHF, Seevinck PR, van den Berg CAT, Isgum I. Deep MR to CT synthesis using unpaired data. In: Lect Notes Comput Sci. Vol 10557. ; 2017:14-23.

30. Zhu JY, Park T, Isola P, Efros AA. Unpaired image-to-image translation using cycleconsistent adversarial networks. In: Proc IEEE Int Conf Comput Vis. ; 2017:2242-2251.

31. Gong K, Yang J, Larson PEZ, et al. MR-based attenuation correction for brain PET using 3D cycle-consistent adversarial network. IEEE Trans Radiat Plasma Med Sci. Published online 2020:[Epub ahead of print]. 


\section{TABLES}

Table 1: Average regional non-absolute and absolute RCs between model-based and deep learning-based $\mu$-maps, compared to CT-based $\mu$-maps.

\begin{tabular}{|c|c|c|c|c|}
\hline & & $\mu$-mapmR5C-CNNAIR & $\mu$-mapMRDL-CNNAIR & Paired t-test \\
\hline \multirow[b]{2}{*}{ Pelvis } & RC (\%) & $2.95 \pm 2.06$ & $-0.13 \pm 0.96$ & $p<0.001$ \\
\hline & $\begin{array}{l}\text { Absolute RC } \\
\text { (\%) }\end{array}$ & $5.10 \pm 1.41$ & $2.60 \pm 0.63$ & $p<0.001$ \\
\hline \multirow[b]{2}{*}{ Bones } & RC (\%) & $-3.21 \pm 3.41$ & $-3.07 \pm 2.79$ & $p=0.81$ \\
\hline & $\begin{array}{l}\text { Absolute RC } \\
(\%)\end{array}$ & $5.31 \pm 2.45$ & $4.54 \pm 1.91$ & $p=0.08$ \\
\hline \multirow{2}{*}{$\begin{array}{l}\text { Fat-based } \\
\text { soft tissue }\end{array}$} & $\mathrm{RC}(\%)$ & $6.20 \pm 2.46$ & $1.28 \pm 0.79$ & $p<0.001$ \\
\hline & $\begin{array}{l}\text { Absolute RC } \\
(\%)\end{array}$ & $6.76 \pm 2.17$ & $2.57 \pm 0.69$ & $p<0.001$ \\
\hline \multirow{2}{*}{$\begin{array}{l}\text { Water- } \\
\text { based soft } \\
\text { tissue }\end{array}$} & $\mathrm{RC}(\%)$ & $1.20 \pm 2.44$ & $-0.77 \pm 0.96$ & $p<0.001$ \\
\hline & $\begin{array}{l}\text { Absolute RC } \\
(\%)\end{array}$ & $3.20 \pm 1.50$ & $2.02 \pm 0.60$ & $p<0.001$ \\
\hline
\end{tabular}




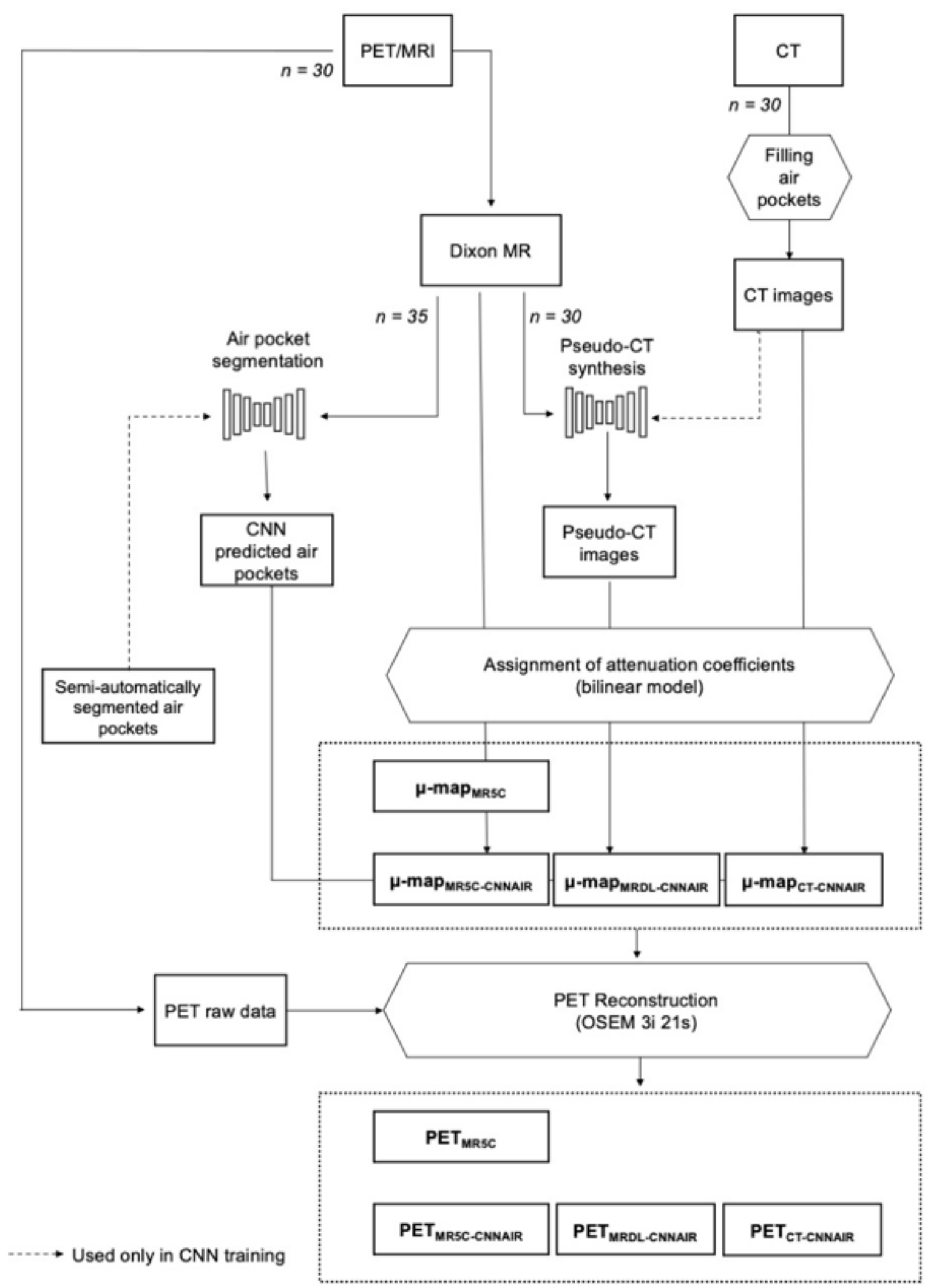

Figure 1: Overview of the methodology. 


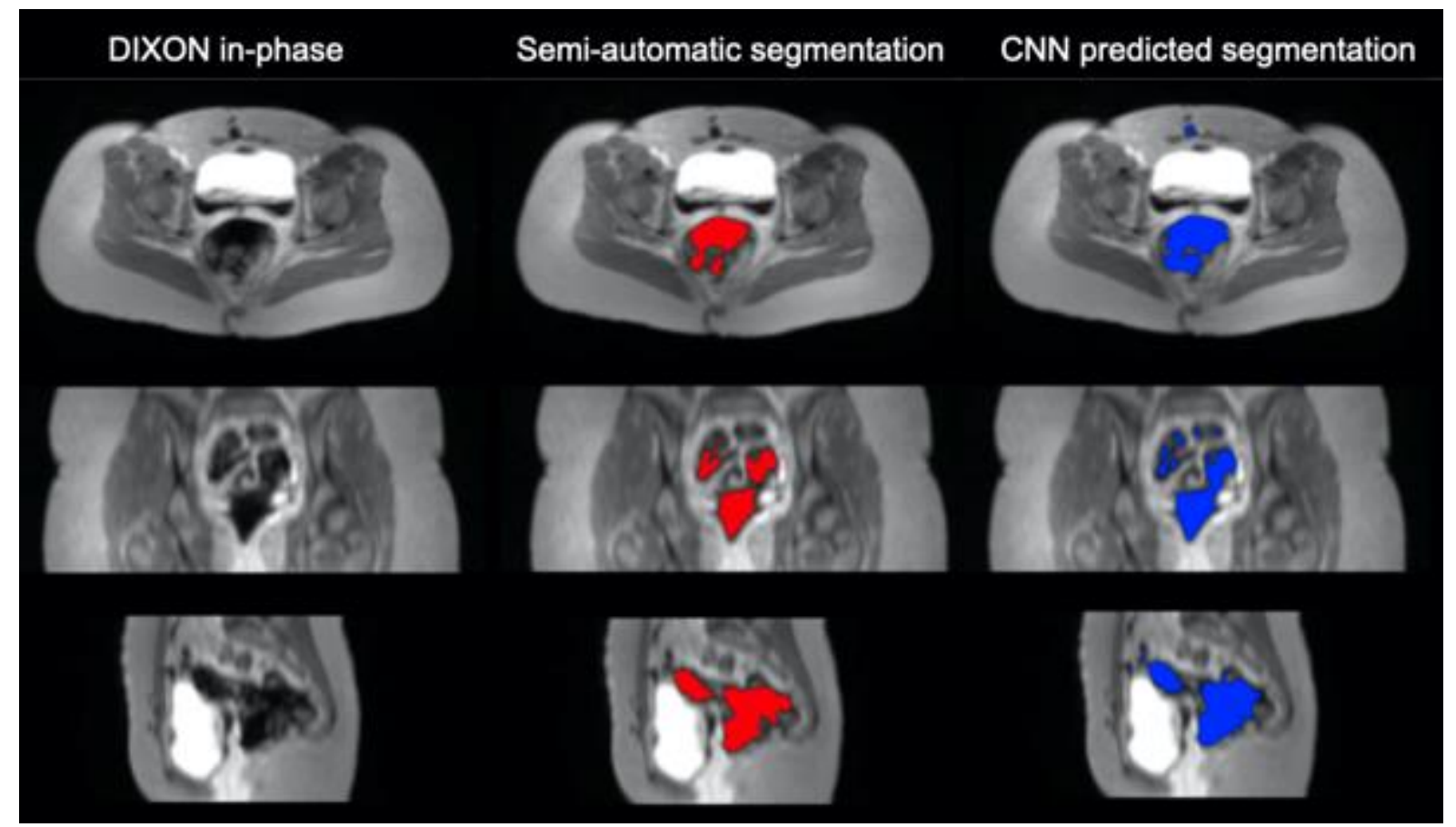

Figure 2: Air pockets segmentation for a representative subject (subject 21). Axial, coronal and sagittal views of the Dixon in-phase MR image are shown with semiautomatic segmentations of the air pockets (red) and segmentation predicted by the CNN (blue). 
A

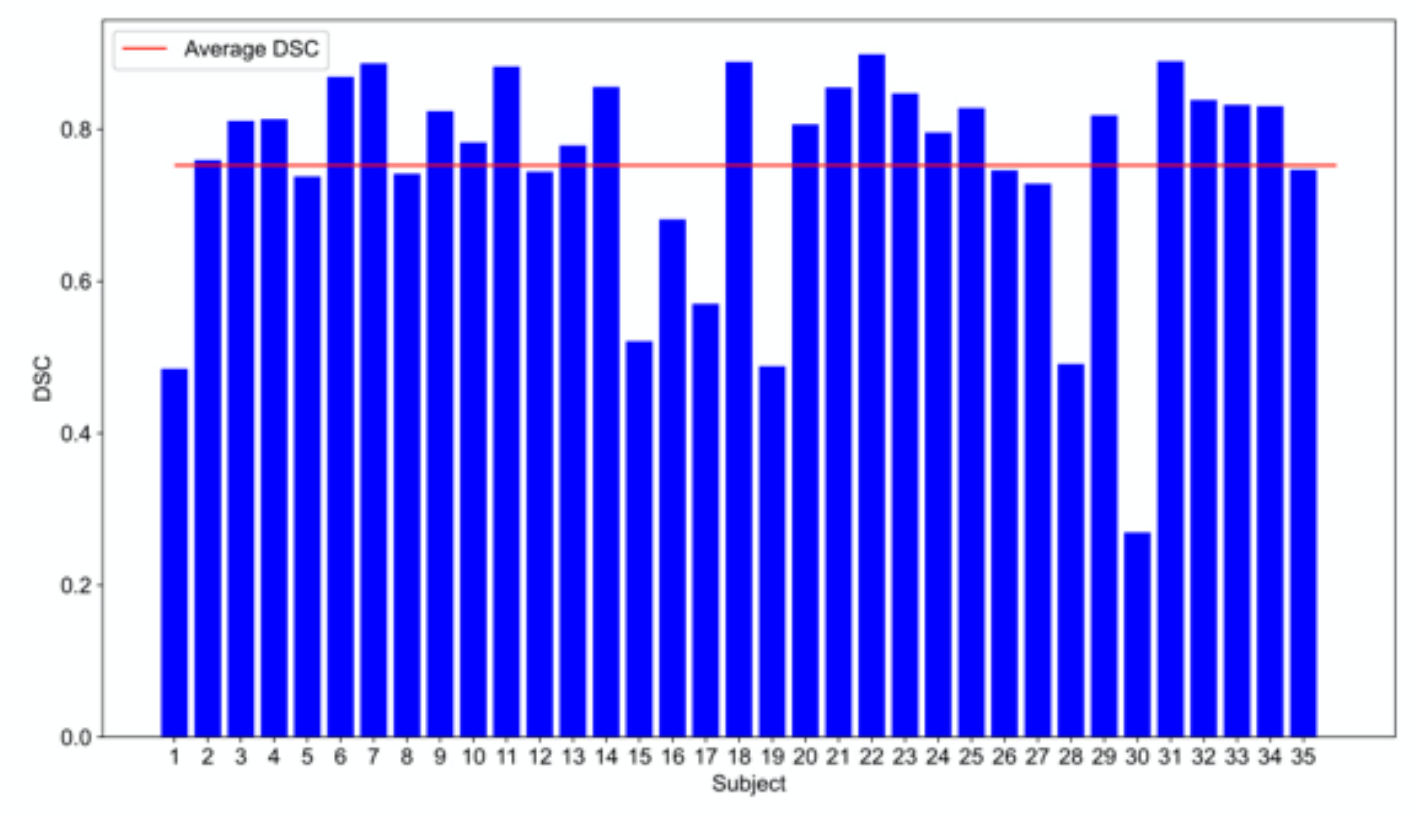

B

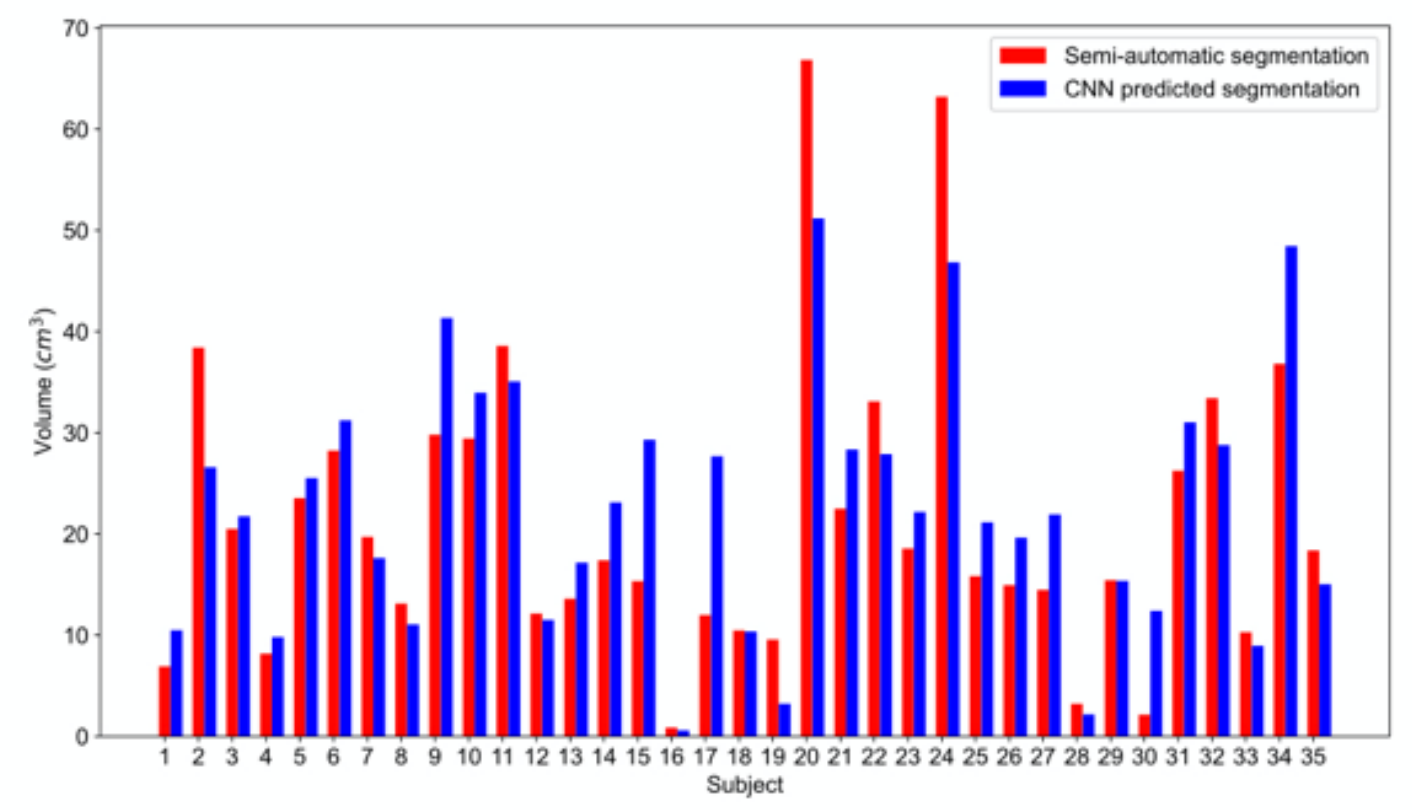

Figure 3: (a) Dice coefficients between the CNN-predicted and semi-automatic segmentations for 35 subjects. The horizontal line represents the mean coefficient. (b) Volume of air in segmented regions obtained using the semi-automatic and the CNN approach. 


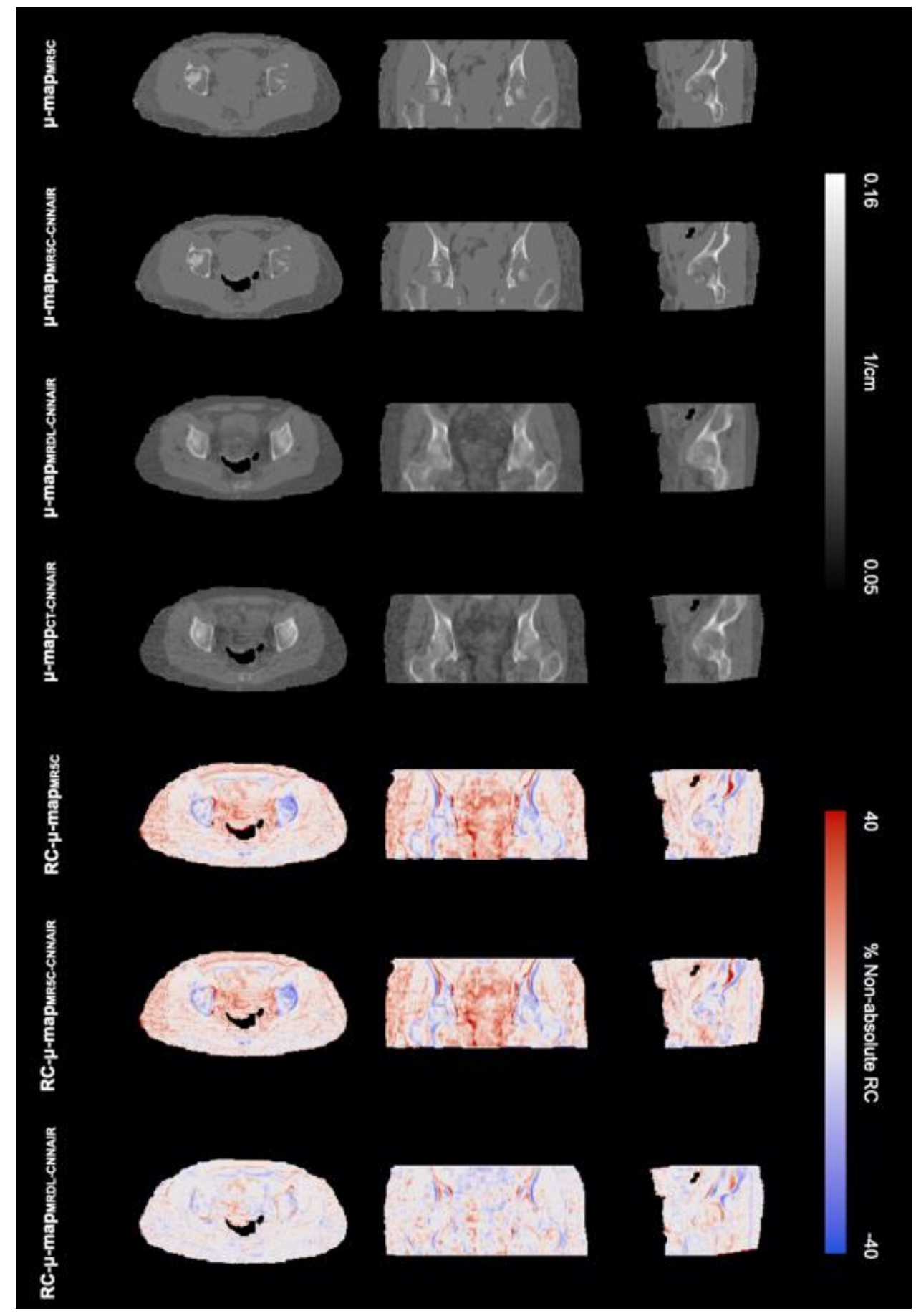

Figure 4: CT- and MR-derived attenuation maps for a representative subject (upper panels). The CT-derived attenuation map with air pockets predicted by the CNN was used as a reference to compute the corresponding relative change maps shown in the lower panels. 


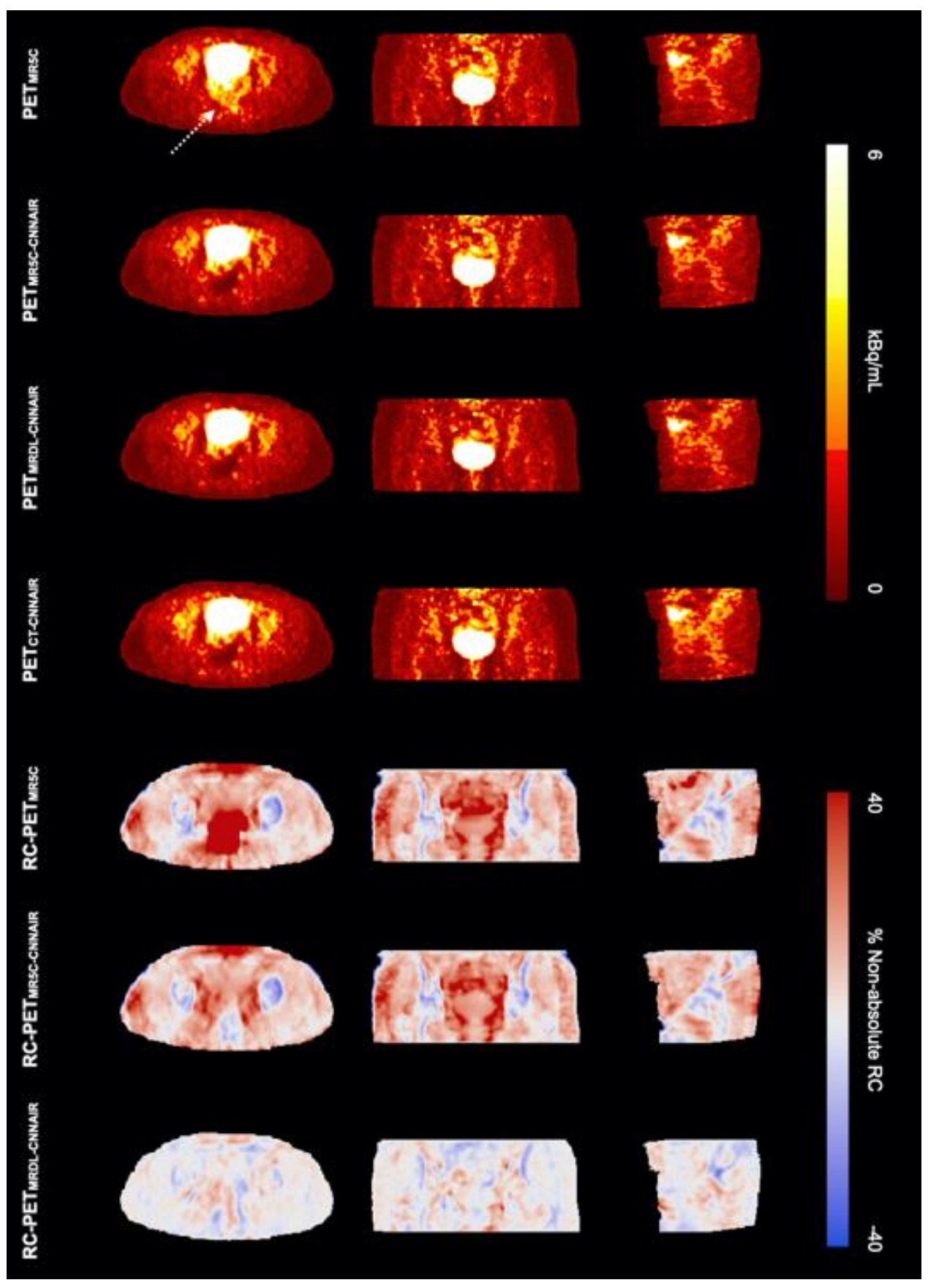

Figure 5: PET images reconstructed using the CT- and MR-based attenuation correction approaches (upper panels). The RC maps for reconstructions performed using each method with respect to the CT-based approach are shown in the lower panels. The arrow indicates an air pocket region which was incorrectly assigned to soft tissue linear attenuation coefficients in $\mu$-mapmR5c. 


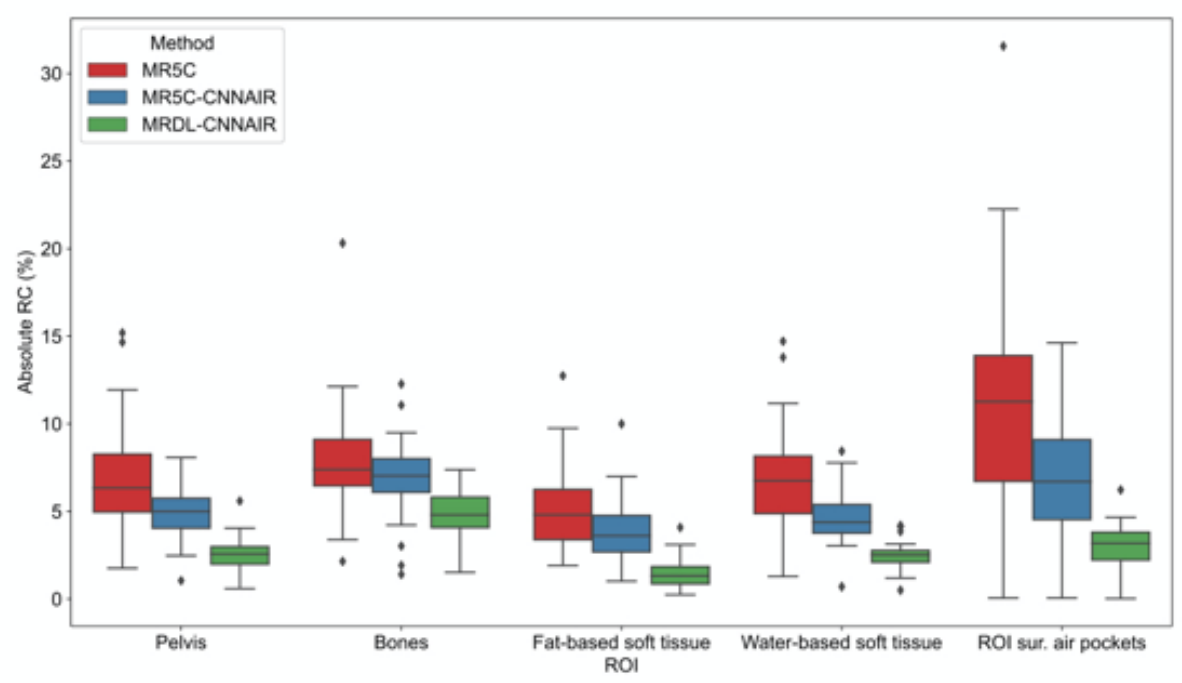

B

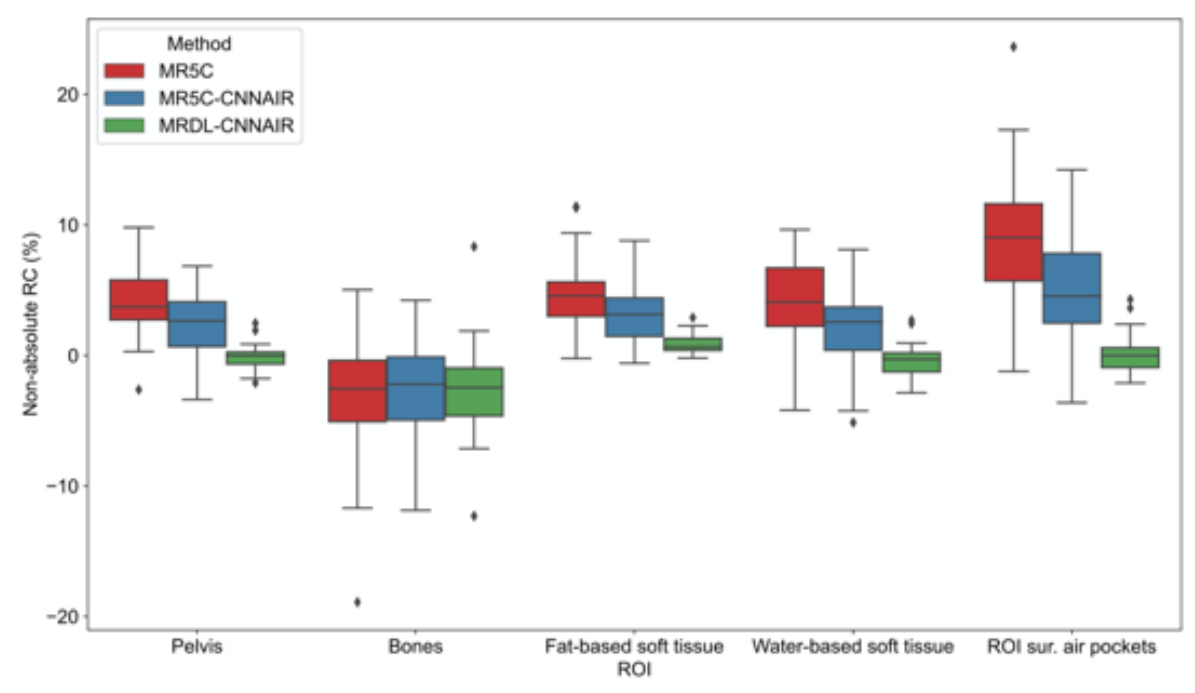

Figure 6: Box plots of (a) absolute and (b) non absolute percentage change in reconstructed PET images using different attenuation correction and air pocket segmentation methods. Box plots were grouped for five ROls. For each box, median is marked using the central horizontal line and edges represent $25^{\text {th }}$ and $75^{\text {th }}$ percentiles of the dataset. Whiskers were determined as 1.5 times the interquartile range, and data points outside this range were identified as outliers. PETCT-CNNAIR was used as reference image in these calculations. 


\section{GRAPHICAL ABSTRACT}

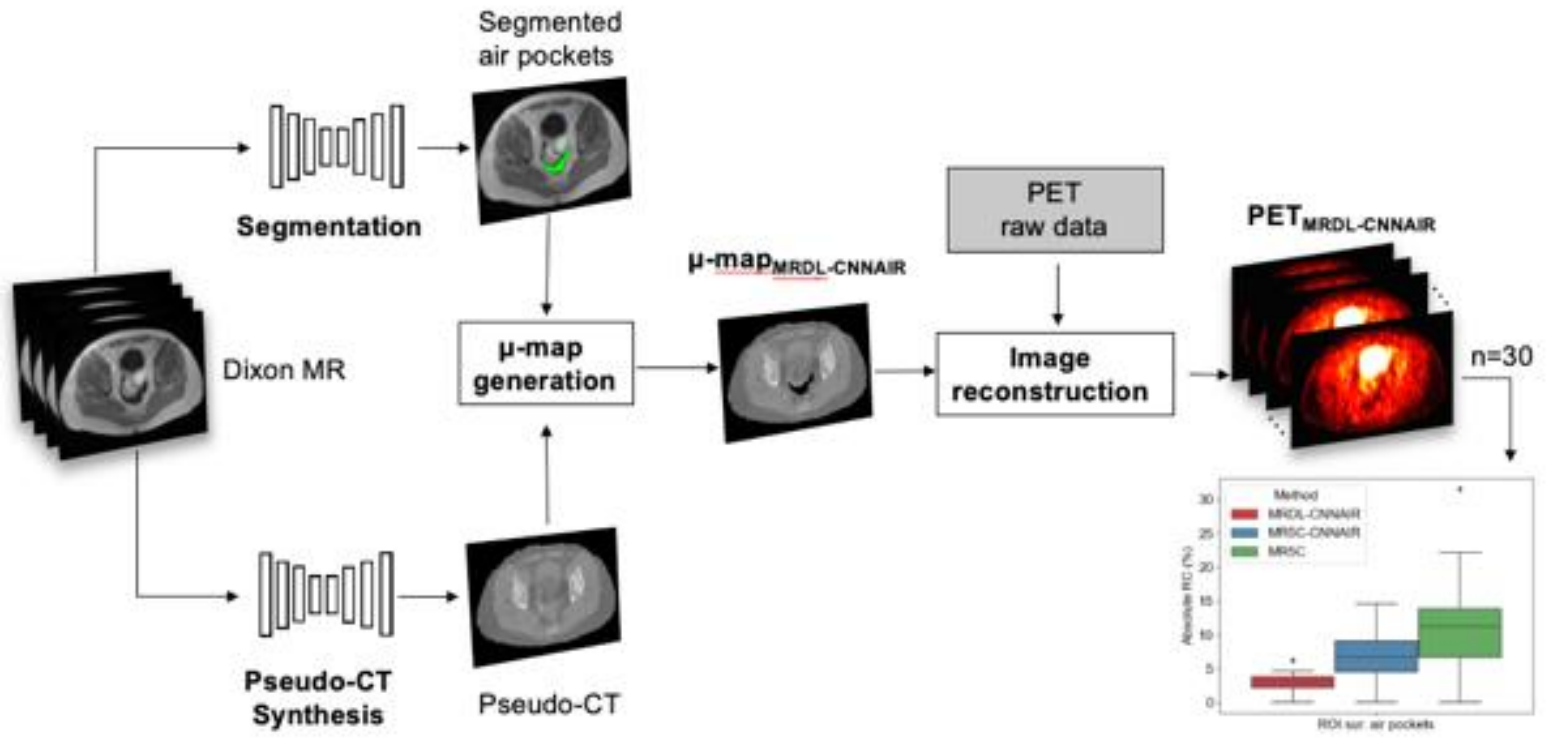




\section{SUPPLEMENTARY FIGURES}

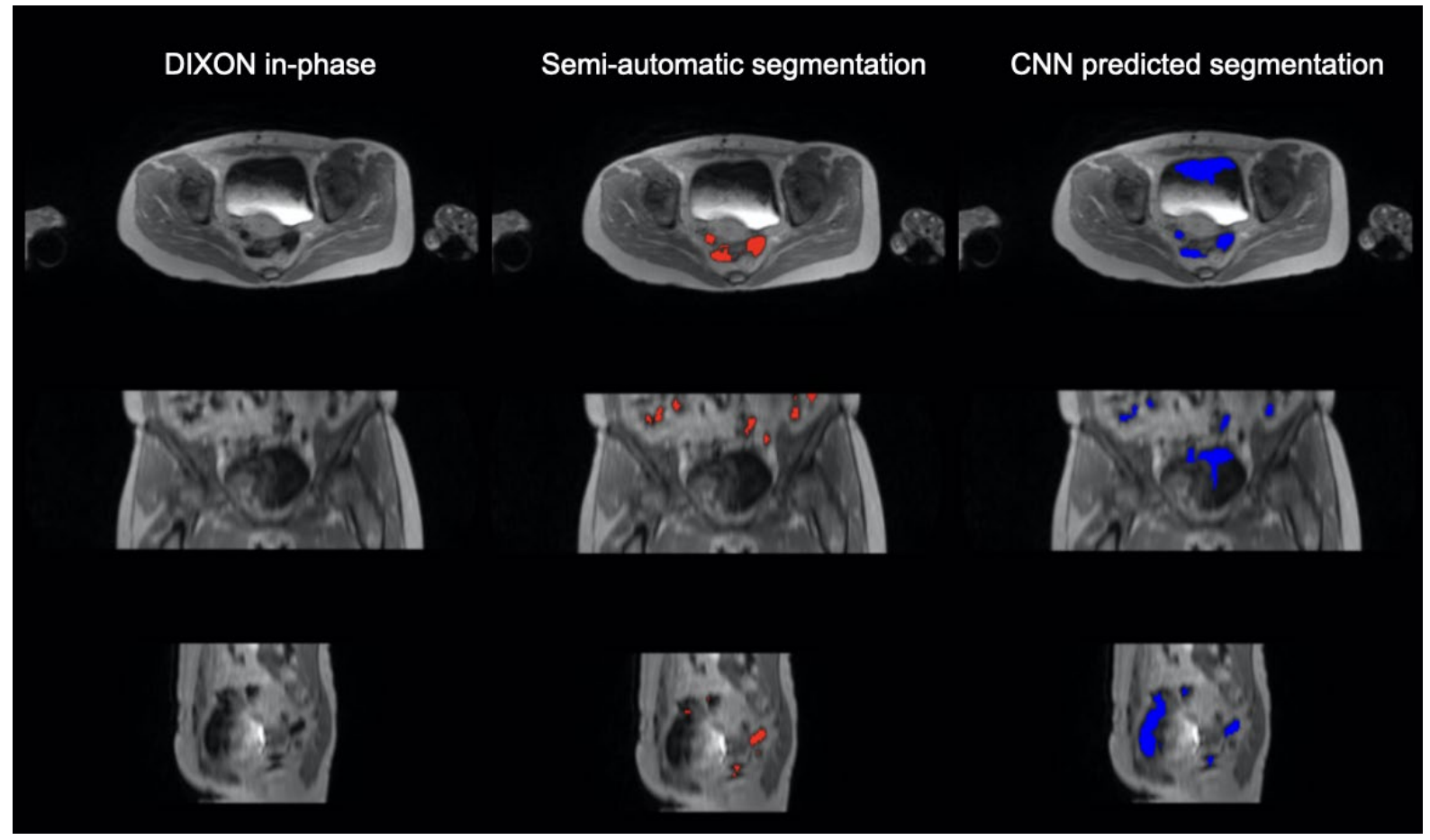

Supplementary Figure 1: Misclassification of bladder voxels in the presence of MR contrast agent. Axial, coronal and sagittal views of the Dixon in-phase MR image, semiautomatic segmentations of the air pockets (red voxels) and segmentation predicted by the CNN (blue voxels) for one representative dataset (subject 1). In this scan, half of the bladder was dark on the MR DIXON images while the remaining half towards the back of the patient had high signal. 


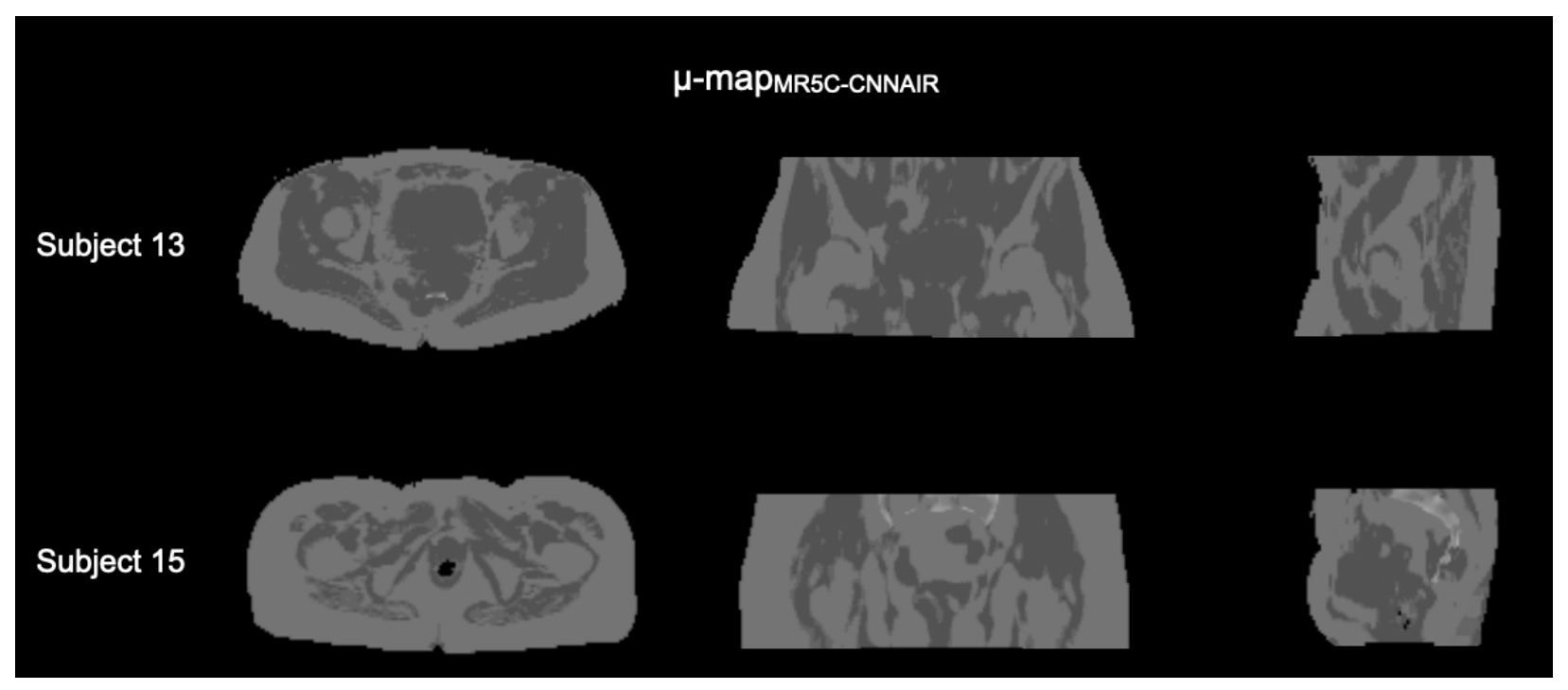

Supplementary Figure 2: Model derived attenuation maps of two subjects where assignment of linear attenuation coefficients has failed in majority of bone structures. 